\title{
HYBRID FEM/BEM FOR HUMAN HEADS EXPOSED TO HIGH FREQUENCY ELECTROMAGNETIC RADIATION
}

\author{
HRVOJE DODIG $^{1}$, MARIO CVETKOVIĆ ${ }^{2}$, DRAGAN POLJAK ${ }^{2}$, AKIMASA HIRATA $^{3}$, ILKKA LAAKSO ${ }^{4}$ \\ ${ }^{1}$ Faculty of Maritime Studies, University of Split, Croatia \\ ${ }^{2}$ Faculty of Electrical Engineering, Mechanical Engineering and Naval Architecture, University of Split, Croatia \\ ${ }^{3}$ Department of Computer Science and Engineering, Nagoya Institute of Technology, Japan \\ ${ }^{4}$ Department of Electrical Engineering and Automation, Aalto University, Finland
}

\begin{abstract}
In this paper the hybrid finite element/boundary element method (FEM/BEM) is used to analyze the human head exposed to plane wave radiation. The radar cross-section (RCS) of a coated sphere at interior resonance frequency obtained using the proposed method showed an excellent agreement with the analytical Mie series solution, thus verifying the validity of the formulation. High frequency electromagnetic wave incident on the human head representing an unbounded scattering problem is formulated via the Stratton-Chu expression, while the interior domain is governed by the vector Helmholtz equation. Some computational examples for the induced electric field are presented. Numerical results showed the highest values of the induced fields around the ocular region and the nose. The presented method is found to be useful in the assessment of the induced fields in the anatomically realistic human models.

Keywords: boundary element method, finite element method, hybrid method, human exposure to electromagnetic fields, human head.
\end{abstract}

\section{INTRODUCTION}

The electromagnetic fields generated by the nowadays ubiquitous sources such as mobile phones, base stations, wireless transmitters, and other telecommunication equipment, have lead to an increasing concern related to the adverse health effects. In order to quantify the induced physical quantities due to the electromagnetic (EM) radiation, it is necessary to establish the link between the external unperturbed EM field and the evaluation of induced physical quantities within the body. This is the main goal of the computational dosimetry, since direct measurements of these quantities are difficult, if even possible.

There are many papers related to high frequency (HF) electromagnetic dosimetry [1-5]. Computational models can be roughly classified as either realistic models (usually obtained using magnetic resonance imaging), or computationally appreciably less demanding, but simplified models, providing the rapid assessment of a quantity of interest. It is usually desirable to have a high resolution anatomically based computational model, to provide accurate results in most of the exposure scenarios [6].

Modern realistic, anatomically based computational models are related to the use of the Finite Difference Time Domain (FDTD) method or the Finite Element Method (FEM), applied to the structured meshes comprising of cubical cells or voxels. The conformal FEM, Boundary Element Method (BEM), and Method of Moments (MoM) are, on the other hand, being used to a somewhat less frequent extent.

Unfortunately, the emergence of commercially available software based on these methods, during the last two decades, has intensified the debate of the superiority of one method over the other, thus obviously clouding the fact that there is no method amenable to all problems and, what is more important, that each method has some inherent advantages and drawbacks, respectively. For example, FDTD has the advantage of simplicity and efficiency in 
analyzing heterogeneous domains, but it suffers from the stair-casing errors related to curved geometries and the inconvenient way in which the computational regions have to be truncated.

FEM, on the other hand, is well suited for modeling complex objects, allowing accurate geometrical representation of structures with curved surfaces, while also allowing elementwise variation in dielectric properties. However, FEM also often requires whole space discretization and the use of an absorbing boundary conditions. Moreover, FEM requires rather large computational resources when finer discretization is used.

The advantage of the conformal methods, such as BEM and MoM is the accurate representation of the domain boundary, avoiding the so called stair-casing effect due to voxel elements. Furthermore, using the Green integral representation, these methods are suitable for the exact treatment of open boundary problems such as human head exposed to incident EM field [7, 8]. However, MoM and BEM are not well suited for modeling complex and arbitrary shaped dielectric objects. Also, numerical implementation results in dense matrices being computationally far more expensive than is the case when FDTD and FEM approaches are used.

The advantages of each of the mentioned numerical techniques can be exploited, leading to an accurate, highly efficient and robust numerical method for addressing various problems, if one does an additional effort in coupling them into hybrid methods. The hybrid method, combining the ability of FEM to deal with arbitrary material properties, and to accurately model curved geometries, and the BEM suitability for the open boundary problems, has been proved to be highly desirable for the accurate characterization of the biological interactions of EMFs [5] compared to the already existing and well-established approaches [7,8].

This paper presents the hybrid finite element/boundary element method (FEM/BEM) approach [9] to the human head exposed to high frequency (HF) electromagnetic radiation. Thus, the present work extends the approach presented in [9] applied to the 3D realistic model of the human eye. The paper is organized as follows. In the first part the Stratton-Chu integral formulation and the vector Helmholtz equation scheme for the human head exposure are given, followed by verification of the method and the description of the human head model. The numerical results for the electric field induced in the head model due to EM plane wave of $900 \mathrm{MHz}$ and $1800 \mathrm{MHz}$, respectively, are presented in the following section. Finally, the conclusion is given in the final part.

\subsection{Hybrid FEM/BEM formulation}

\section{THEORETICAL BACKGROUND}

Electromagnetic wave incident on the human head can be treated as unbounded scattering problem. Using the Stratton-Chu integral formulation, the time harmonic electric field in the domain exterior to the head is expressed by the following boundary integral equation $[5,9]$ :

$$
\begin{aligned}
& \alpha \vec{E}_{\text {ext }}^{\prime}=\vec{E}_{i n c}^{\prime}+\oint_{\partial V} \hat{n} \times\left(\nabla \times \vec{E}_{\text {ext }}\right) G d S+ \\
& \oint_{\partial V}\left[\left(\hat{n} \times \vec{E}_{\text {ext }}\right) \times \nabla G+\left(\hat{n} \cdot \vec{E}_{\text {ext }}\right) \nabla G\right] d S,
\end{aligned}
$$

where $\hat{n}$ is an outer normal to surface $\partial V$ bounding the volume $V$ and $\alpha$ is the solid angle subtended at the observation point. $\vec{E}_{e x t}$ and $\vec{E}_{i n c}$ denote the total and the incident electric field, respectively, while $\mathrm{G}$ is the free space Green's function given by:

$$
G=G\left(\vec{r}, \vec{r}^{\prime}\right)=\frac{e^{-j k R}}{4 \pi R} ; \quad R=\left|\vec{r}-\vec{r}^{\prime}\right|
$$


where $R$ is the distance from the observation point $\vec{r}$ to the source point $\vec{r}^{\prime}$, and $k$ is a wave number.

Performing some manipulations, (1) can be specified in terms of tangential components of electric field $\vec{E}$ and magnetic field $\vec{H}$ on the boundary surface $\partial V$ :

$$
\begin{aligned}
\alpha \vec{E}_{e x t}^{\prime}=\vec{E}_{i n c}^{\prime}- & j \omega \mu \oint_{\partial V} \hat{n} \times \vec{H}_{e x t} G d S+ \\
& +\oint_{\partial V}\left[\left(\hat{n} \times \vec{E}_{e x t}\right) \times \nabla G-\frac{1}{\sigma+j \omega \mu} \nabla_{s} \cdot\left(\hat{n} \times \vec{H}_{e x t}\right) \nabla G\right] d S .
\end{aligned}
$$

Eqn (3) is of appropriate form pertinent for coupling to the governing differential equations for the interior inhomogeneous region, given by:

$$
\nabla \times\left(\frac{j}{\omega \mu} \nabla \times \vec{E}_{i n t}\right)-(\sigma+j \omega \varepsilon) \vec{E}_{\text {int }}=0 .
$$

The fields $\vec{E}$ and $\vec{H}$ are approximated using the edge elements [10] that preserve the tangential continuity of the fields on the boundary

$$
\begin{aligned}
& \vec{E}=\sum_{i=1}^{n} \delta_{i} \vec{w}_{i} e_{i}, \\
& \vec{H}=\sum_{i=1}^{n} \delta_{i} \vec{w}_{i} h_{i} .
\end{aligned}
$$

The unknown coefficients $e_{i}$ and $h_{i}$, respectively, associated with each edge of the model, are determined from the global system of equations. The coefficient $\delta_{i}= \pm 1$ is equal to 1 if the local edge direction coincides with chosen global edge direction, otherwise it is -1 .

Vector base shape function $w_{k}$ is given by [11]:

$$
\vec{w}_{k}=N_{i} \nabla N_{j}-N_{j} \nabla N_{i},
$$

where $N_{i}$ and $N_{j}$ are regular first order barycentric shape functions.

Applying the weighted residual approach to (4), by taking the dot product of (4) with test function $w_{i}$, and using the Galerkin-Bubnov procedure, it can be written

$$
\int_{V} \delta_{i} \vec{w}_{i} \cdot\left[\nabla \times\left(\frac{j}{\omega \mu} \nabla \times \vec{E}_{i n t}\right)-(\sigma+j \omega \varepsilon) \vec{E}_{i n t}\right] d V=0 .
$$

After applying some standard vector identities, followed by the divergence theorem, the weak form is obtained:

$$
\int_{V}\left[\frac{j}{\omega \mu} \nabla \times \delta_{i} \vec{w}_{i} \cdot \vec{E}_{i n t}-(\sigma+j \omega \varepsilon) \delta_{i} \vec{w}_{i} \cdot \vec{E}_{i n t}\right] d V=\oint_{\partial V} \overrightarrow{d S} \cdot \delta_{i} \vec{w}_{i} \times \vec{H}_{\text {int }} .
$$

Now FEM/BEM coupling can be employed by exploiting the fact that tangential components of electric and magnetic fields must be continuous across the surface $\partial \mathrm{V}$. Because of the tangential continuity one has $\hat{n} \times \vec{E}_{i n t}=\hat{n} \times \vec{E}_{\text {ext }}$ and $\hat{n} \times \vec{H}_{\text {int }}=\hat{n} \times$ $\vec{H}_{\text {ext }}$. Thus, it can be written

$$
\oint_{\partial V} \overrightarrow{d S}^{\prime} \cdot \delta_{i} \vec{w}_{i} \times \vec{E}_{i n t}^{\prime}=\oint_{\partial V} \overrightarrow{d S}^{\prime} \cdot \delta_{i} \vec{w}_{i} \times \vec{E}_{e x t}^{\prime}
$$


One can now substitute $\vec{E}_{\text {ext }}$ and $\vec{H}_{\text {ext }}$ in (3) with $\vec{E}_{\text {int }}$ and $\vec{H}_{i n t}$, respectively, and after inserting (3) into (10), the following double surface integral is obtained:

$$
\begin{aligned}
\oint_{\partial V} \overrightarrow{d S}^{\prime} \cdot \delta_{i} \vec{w}_{i} \times \alpha \vec{E}_{e x t}^{\prime} & =\oint_{\partial V} \overrightarrow{d S^{\prime}} \cdot \delta_{i} \vec{w}_{i} \times \vec{E}_{i n c}^{\prime}- \\
& \quad-j \omega \mu \oint_{\partial V} \overrightarrow{d S}^{\prime} \cdot \delta_{i} \vec{w}_{i} \times \oint_{\partial V} \hat{n} \times \vec{H}_{i n t} G d S+ \\
& +\oint_{\partial V} \overrightarrow{d S}^{\prime} \cdot \delta_{i} \vec{w}_{i} \times \oint_{\partial V}\left(\hat{n} \times \vec{E}_{i n t}\right) \times \nabla G d S- \\
& \quad-\frac{1}{\omega+j \omega \mu} \oint_{\partial V} \overrightarrow{d S}^{\prime} \cdot \delta_{i} \vec{w}_{i} \times \oint_{\partial V} \nabla_{s} \cdot\left(\hat{n} \times \vec{H}_{e x t}\right) \nabla G d S .
\end{aligned}
$$

Inserting (5) and (6) into (11) produces the system of equations related to edges at bounding surface of the problem:

$$
\begin{aligned}
{\left[E_{\text {bem }}\right]\left\{e_{\text {bem }}\right\} } & =\left\{e_{\text {inc }}\right\}+\left[H_{\text {bem }}\right]\left\{h_{\text {bem }}\right\}, \\
{\left[E_{\text {fem }}\right]\left\{e_{\text {fem }}\right\} } & =\left[H_{\text {fem }}\right]\left\{h_{\text {fem }}\right\},
\end{aligned}
$$

where $e_{\text {bem }}$ and $h_{\text {bem }}$ are unknown coefficients associated with boundary surface of the scattering problem, $e_{i n c}$ are known coefficients calculated from the incident field, and matrices $\left[E_{b e m}\right]$ and $\left[H_{b e m}\right]$ arise from boundary integral eqn (11), while matrices $\left[E_{f e m}\right]$ and $\left[H_{f e m}\right]$ stem from FEM eqn (9).

\subsection{Verification of the hybrid method}

In order to verify the accuracy of the proposed hybrid formulation, first the results for the bistatic radar cross-section (RCS), an important parameter characterizing the EM scattering, are presented. The RCS of a coated metallic sphere at $300 \mathrm{MHz}$, corresponding to interior

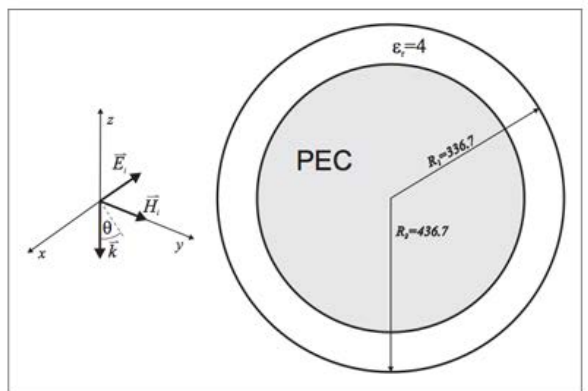

a)

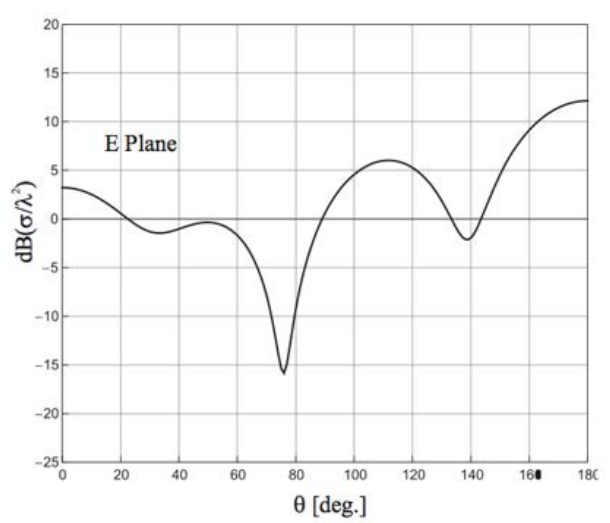

b)

Figure 1: Bistatic RCS of a metallic sphere coated with dielectric layer of $\varepsilon_{r}=4$ at 300 MHz. (a) Geometry of the problem. (b) Results obtained using hybrid FEM/BEM formulation and analytical solution based on Mie theory from [12]. 

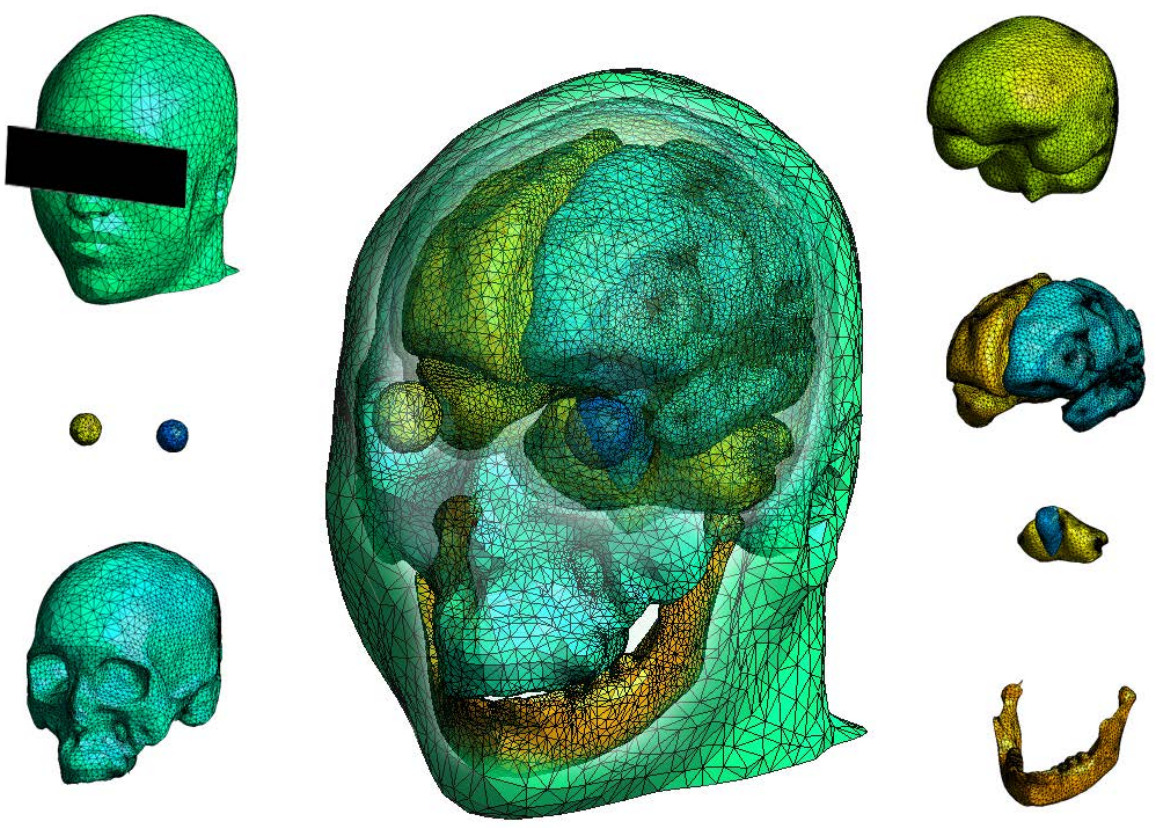

Figure 2: Model of the human head with insets showing different subdomains (tissues).
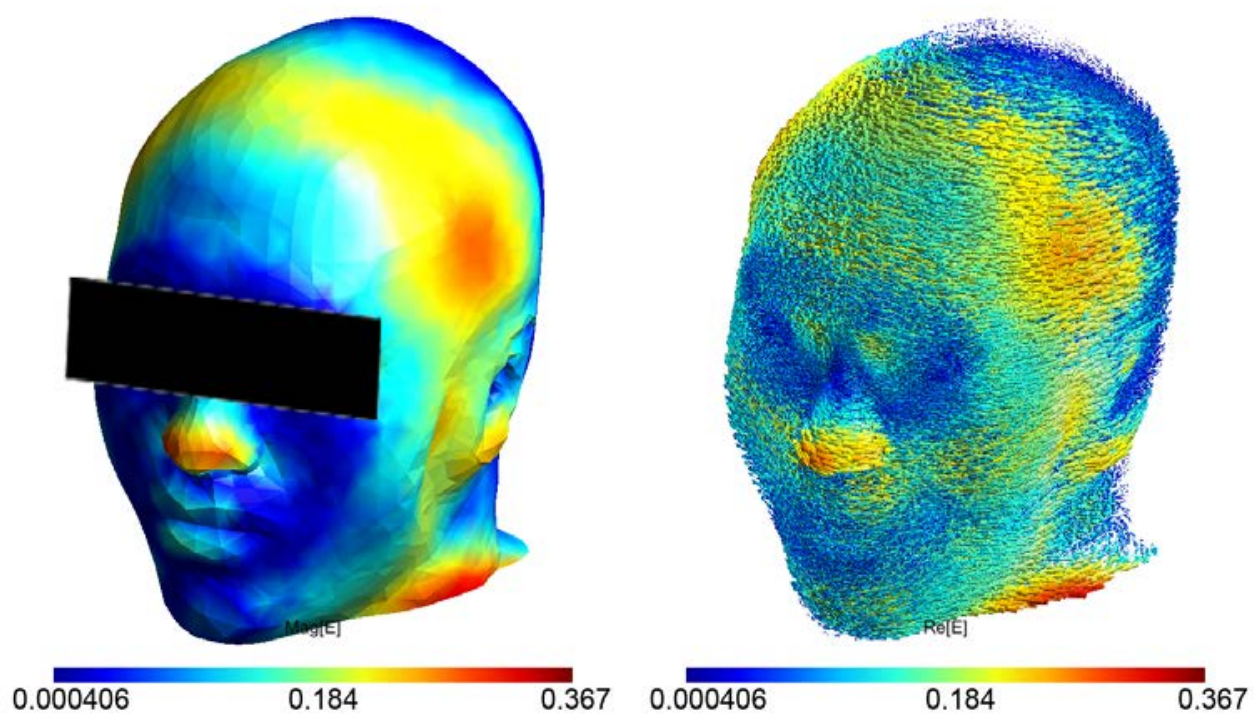

Figure 3: Electric field induced on the surface of the human head model due to $900 \mathrm{MHz}$ horizontally polarized plane wave (left). Direction of the electric field (right). 


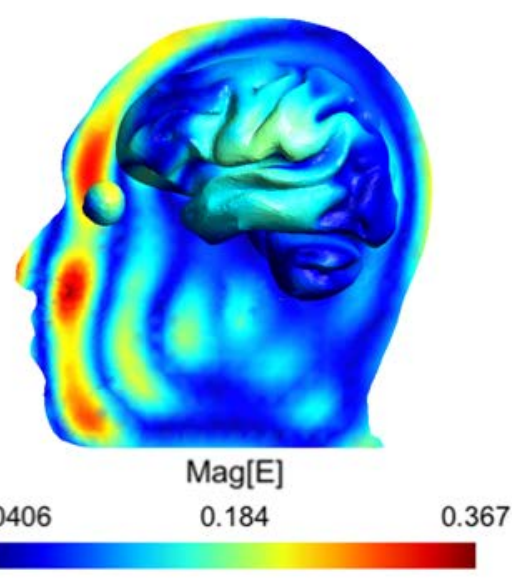

a)

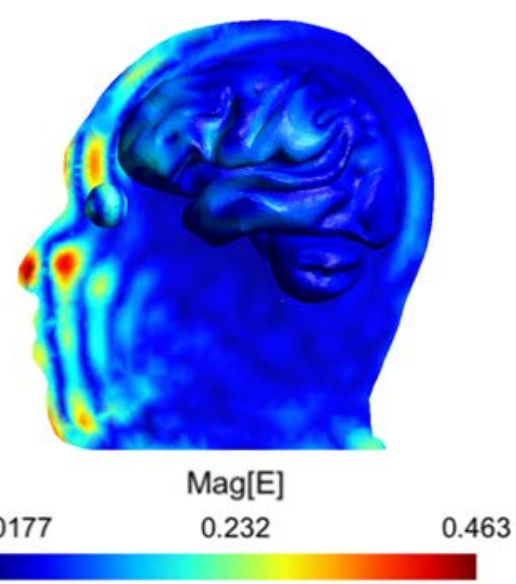

c)

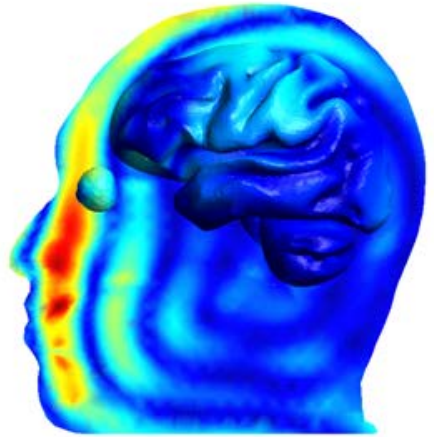

$\operatorname{Mag}[E]$

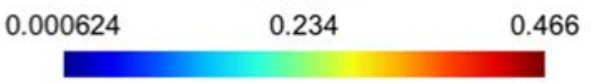

b)

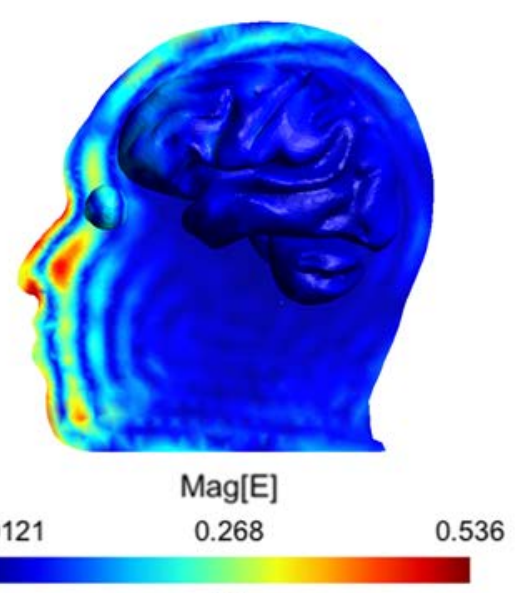

d)

Figure 4: Induced electric field in the sagittal cross-section of the head due to: (a) $900 \mathrm{MHz}$ HP, (b) $900 \mathrm{MHz}$ VP, (c) $1800 \mathrm{MHz} \mathrm{HP}$, (d) $1800 \mathrm{MHz}$ VP.

resonance frequency, is shown in Fig. 1(a). The comparison of the FEM/BEM results with the analytical solution, obtained using the Mie theory [12], showed excellent agreement, as shown in Fig. 1(b)

\subsection{The human head model}

The model of the human head including the various head tissues is shown in Fig. 2. The model was constructed from magnetic resonance imaging (MRI) of a 24-year old male [13]. The current implementation of model consists of 8 tissues, whose frequency dependent dielectric parameters, modeled using the 4-Cole-Cole method [14] are given in Table 1. The boundary surface of the head model is discretized using 6.838 triangular elements, while interior domain of the head is discretized using 1.034.641 tetrahedral elements. 


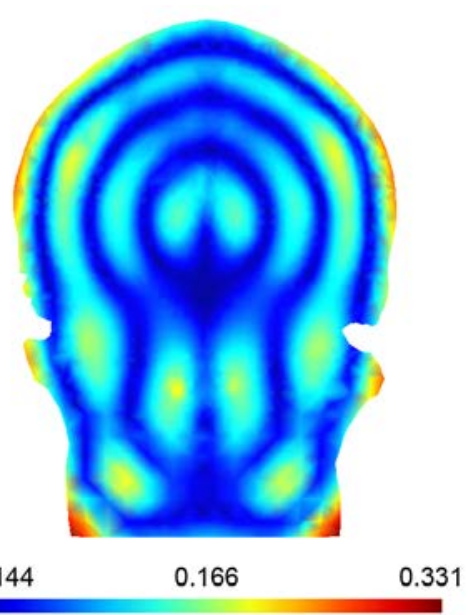

a)

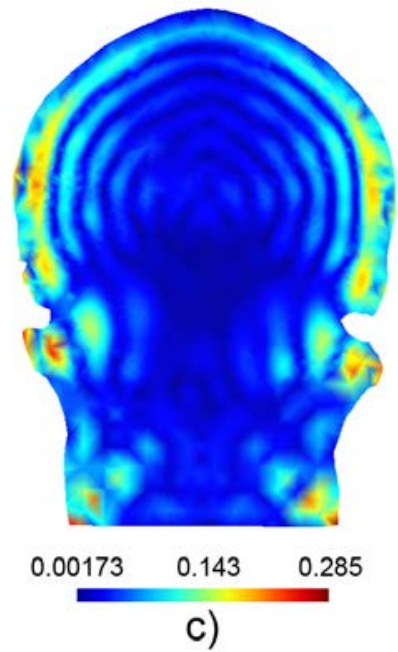

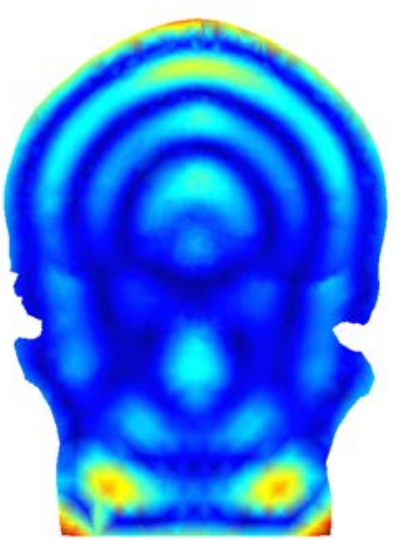

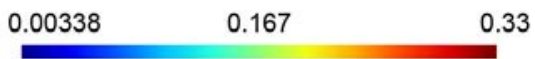

b)

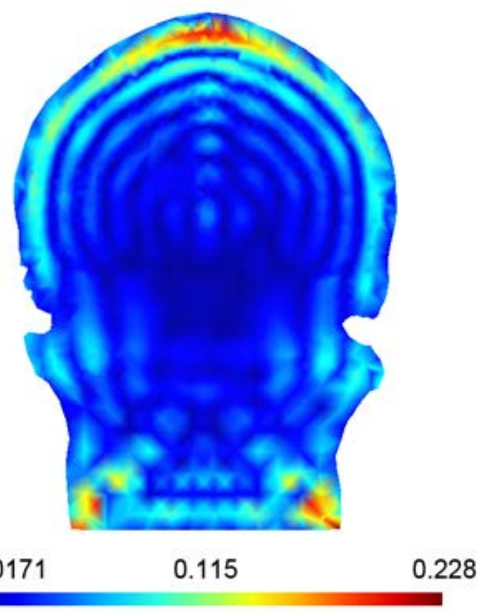

d)

Figure 5: Induced electric field in the coronal cross-section of the head due to: (a) $900 \mathrm{MHz}$ HP, (b) $900 \mathrm{MHz}$ VP, (c) $1800 \mathrm{MHz} \mathrm{HP}$, (d) $1800 \mathrm{MHz}$ VP.

Table 1: Tissue dielectric parameters according to the 4-Cole-Cole Model described in [14].

\begin{tabular}{lllll}
\hline & \multicolumn{2}{l}{$900 \mathrm{MHz}$} & \multicolumn{2}{l}{$1800 \mathrm{MHz}$} \\
\hline Tissue & $\sigma(\mathrm{S} / \mathrm{m})$ & $\varepsilon(-)$ & $\sigma(\mathrm{S} / \mathrm{m})$ & $\varepsilon(-)$ \\
\hline \hline Brainstem & 0.591 & 38.886 & 0.915 & 37.011 \\
Cerebellum & 1.263 & 49.444 & 1.709 & 46.114 \\
Eye (vitreous) & 1.636 & 68.902 & 2.032 & 68.573 \\
Head skin & 0.867 & 41.405 & 1.185 & 38.872 \\
Skull and mandible & 0.339 & 20.788 & 0.588 & 19.343 \\
Grey matter & 0.942 & 52.725 & 1.391 & 50.079 \\
Muscle tissue & 0.943 & 55.032 & 1.341 & 53.549 \\
\hline
\end{tabular}



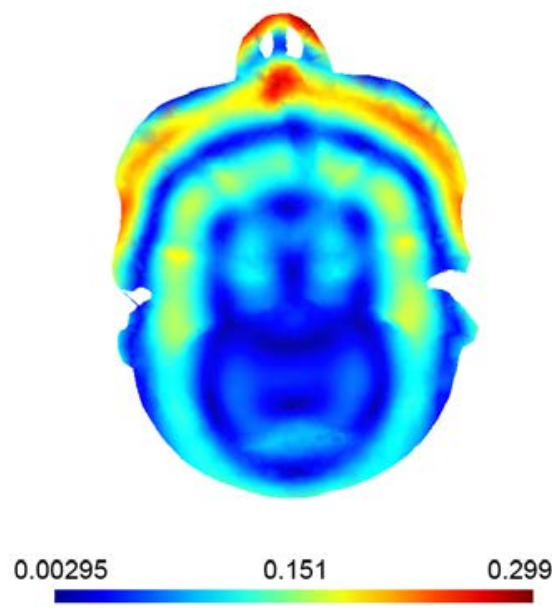

a)

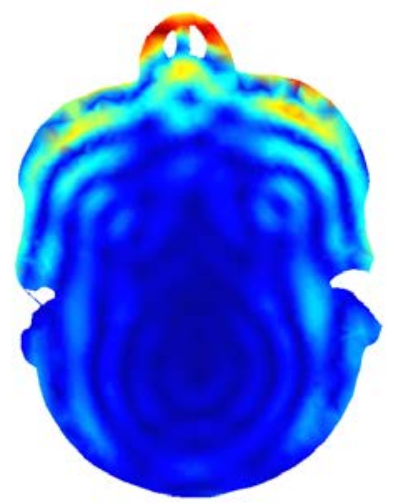

0.231

c)
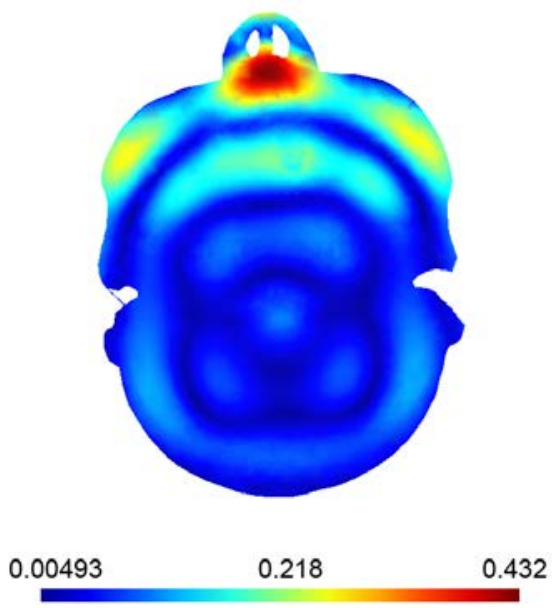

b)

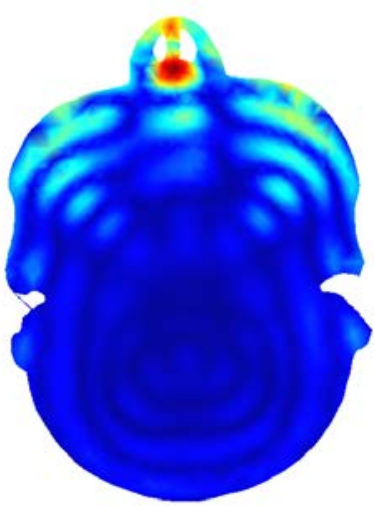

0.215

d)

Figure 6: Induced electric field in the transverse cross-section of the head due to: (a) 900 MHz HP, (b) 900 MHz VP, (c) 1800 MHz HP, (d) 1800 MHz VP.

\section{NUMERICAL RESULTS}

The computational examples are given for the human head exposed to incident plane wave of $900 \mathrm{MHz}$ and $1800 \mathrm{MHz}$. Incident field $\vec{E}_{i n c}=1 \mathrm{~V} / \mathrm{m}$ is horizontally and vertically polarized, and directed toward the nose, perpendicular to the coronal head crosssection. Numerical results for the horizontal polarization are denoted by HP, while vertical polarization is denoted by VP.

The illustrative results for the electric field induced on the surface of the head model are shown in Fig. 3 for $900 \mathrm{MHz}$ horizontally polarized EM wave. The highest value of the field is obtained at the tip of the nose. 
Figs 4-6 show the results for the induced field on the sagittal, coronal, and transverse cross-sections of the head, respectively, due to $900 \mathrm{MHz}$ and $1800 \mathrm{MHz}$, vertically, i.e. horizontally polarized EM wave.

As evident from Figs 4 and 6, the highest values of the induced field are formed in regions around the nose and the eyes, suggesting possible formation of hot-spots. Further dosimetry calculations, related to estimation of the specific absorption rate (SAR) and the related temperature increase, are currently under way, since according to international guidelines [15], the temperature elevation in the human eye is listed as one of the most important concern, as well as in the brain. Furthermore, inclusion of the detailed geometrical eye model, consisting of various ocular tissues, as reported in $[6,9,16]$, could provide additional insight into related thermal effects.

Finally, considering the influence of the polarization of the incident electromagnetic wave on the induced field, the results show somewhat higher values for the vertical polarization. The study in [17] attributed this fact to the component of the surface area perpendicular to the incident electric field. The results obtained in this work suggest the same to be the case.

\section{CONCLUSION}

The paper presents the application of the hybrid FEM/BEM formulation to the analysis of the electric field induced in the human head due to exposure to HF plane wave of different polarizations. The boundary integral expression provides an efficient treatment of unbounded region exterior to the head, thus, avoiding the use of absorbing boundary conditions. On the other hand, FEM enables efficient treatment of the head interior regions. As both implemented methods employ conformal meshes - BEM for problem boundary, and FEM for interior boundaries between different regions, respectively - detailed geometry of head tissues can be taken into account, thus overcoming potential undesired stair-casing effect occurring when rectilinear meshes and voxels are used. Further comparison between the proposed method and other already established methods such as FDTD and FEM will be carried out in the future work.

\section{REFERENCES}

[1] Hand, J., Modelling the interaction of electromagnetic fields (10 mhz-10 ghz) with the human body: methods and applications. Physics in medicine and biology, 53(16), p. R243, 2008.

[2] Poljak, D., Electromagnetic fields: Environmental exposure. Encyclopedia of Environmental Health, Elsevier, 2011.

[3] Singh, K.D., Logan, N.S. \& Gilmartin, B., Three-dimensional modeling of the human eye based on magnetic resonance imaging. Investigative Ophthalmology \& Visual Science, 47(6), pp. 2272-2279, 2006.

[4] Hirata, A., Temperature increase in human eyes due to near-field and far-field exposures at $900 \mathrm{mhz}, 1.5 \mathrm{ghz}$, and $1.9 \mathrm{ghz}$. IEEE Transactions on Electromagnetic Compatibility, 47(1), pp. 68-76, 2005.

[5] Poljak, D., Cavka, D., Dodig, H., Peratta, C. \& Peratta, A., On the use of the boundary element analysis in bioelectromagnetics. Engineering Analysis with Boundary Elements, 49, pp. 2-14, 2014.

[6] Poljak, D., Cvetković, M., Dodig, H. \& Peratta, A., Electromagnetic-thermal analysis for human exposure to high frequency HF radiation. International Journal of Design \& Nature and Ecodynamics, 12(1), pp. 55-67, 2017. 
[7] Cvetković, M. \& Poljak, D., An efficient integral equation based dosimetry model of the human brain. Proceedings of EMC EUROPE 2014, Gothenburg, Sweden, 1-4 September 2014, pp. 375-380, 2014.

[8] Poljak, D., Cvetković, M., Peratta, A., Peratta, C., Dodig, H. \& Hirata, A., On some integral approaches in EM dosimetry. BIOEM 2016, 2016.

[9] Dodig, H., Poljak, D. \& Peratta, A., Hybrid BEM/FEM edge element computation of the thermal rise in the 3D model of the human eye induced by high frequency EM waves. Software, Telecommunications and Computer Networks (SoftCOM), 2012 20th International Conference on, IEEE, pp. 1-5, 2012.

[10] Nédélec, J.C., Mixed finite elements in $R^{3}$. Numerische Mathematik, 35(3), pp. 315$341,1980$.

[11] Volakis, J.L., Chatterjee, A. \& Kempel, L.C., Finite Element Method Electromagnetics: Antennas, Microwave Circuits, and Scattering Applications, Volume 6. John Wiley \& Sons, 1998.

[12] Ji, Y., Wang, H. \& Hubing, T.H., A numerical investigation of interior resonances in the hybrid FEM/MoM method. IEEE Transactions on Antennas and Propagation, 51(2), pp. 347-349, 2003.

[13] Laakso, I., Tanaka, S., Koyama, S., De Santis, V. \& Hirata, A., Inter-subject variability in electric fields of motor cortical tDCS. Brain Stimulation, 8(5), pp. 906-913, 2015.

[14] Gabriel, C. \& Gabriel, S., Compilation of the dielectric properties of body tissues at $\mathrm{RF}$ and microwave frequencies. Technical report, King's College London, AL/OE-TR1996-0037, 1996.

[15] IEEE, Standard for safety levels with respect to human exposure to radio frequency electromagnetic fields, $3 \mathrm{kHz}$ to $300 \mathrm{GHz}$. IEEE Std C951-2005 (Revision of IEEE Std C951-1991), pp. 1-238, 2006.

[16] Dodig, H., Lalléchère, S., Bonnet, P., Poljak, D. \& Drissi, K.E.K., Stochastic sensitivity of the electromagnetic distributions inside a human eye modeled with a $3 \mathrm{~d}$ hybrid bem/fem edge element method. Engineering Analysis with Boundary Elements, 49, pp. 48-62, 2014.

[17] Hirata, A., Ito, N. \& Fujiwara, O., Influence of electromagnetic polarization on the whole-body averaged SAR in children for plane-wave exposures. Physics in Medicine and Biology, 54(4), p. N59, 2009. 